\title{
Multiplexed MS/MS in a Miniature Rectilinear Ion Trap
}

\author{
Adam M. Graichen, Richard W. Vachet
}

Department of Chemistry, University of Massachusetts, LGRT 701, 710 N. Pleasant St., Amherst, MA, 01003, USA

\begin{abstract}
A multiplexed method for performing MS/MS on multiple ions simultaneously in a miniature rectilinear ion trap (RIT) mass spectrometer has been developed. This method uses an ion encoding procedure that relies on the mass bias that exists when ions are externally injected into an RIT operated with only a single phase if applied to one pair of electrodes. The ion injection profile under such conditions ions is Gaussian-like over a wide range of if amplitudes, or low mass cutoff (LMCO) values, during ion accumulation. We show that this distribution is related to ion $\mathrm{m} / \mathrm{z}$ and is likely caused by ions having an optimal range of pseudo-potential well depths for efficient trapping. Based on this observation, precursor ion intensity changes between two different injection LMCO values can be predicted, and these ion intensity changes are found to be carried through to their corresponding product ions, enabling multiplexed MS/MS spectra to be deconvoluted.
\end{abstract}

Key words: Tandem mass spectrometry, Multiplexed, Portable mass spectrometer, Rectilinear ion trap

\section{Introduction}

$\mathrm{T}$ he interest in miniaturized mass spectrometers has increased as they have found application in a multitude of environments. Applications include monitoring public safety areas [1], detecting chemical warfare agents (CWA) in battlefield situations [2, 3], analyzing harsh environmental conditions $[4,5]$, ensuring industrial quality control [6], and even screening in medicine [7]. One of the shortcomings for in situ analysis is sample collection, extraction, separation, or other special treatments that are needed for certain samples [8]. This weakness is particularly evident in complex mixtures where more detailed or further structural information is desired.

To overcome the ambiguity and difficulty in the analysis of complex mixtures, a common practice is to couple MS with various separation techniques, and this has been done with miniaturized mass spectrometers as well. For instance, there has been much effort recently in coupling scaled-down gas chromatographs (GC) with portable MS systems [2, 3, 9,

Correspondence to: Richard W. Vachet; e-mail: rwvachet@chem.umass.edu
10]. These GC systems have been made more field-portable, and the resulting analysis times have been significantly reduced. This short analysis time, however, inhibits tandem MS data from being collected for many of the species. Numerous injections/analyses may be required to obtain the information needed to truly identify all mixture components.

To avoid many of the difficulties, limitations, and lack of development with portable separation techniques, we are investigating a multiplexed MS/MS approach on a miniature RIT [1, 11, 12] using an ion intensity encoding scheme. In this approach, multiple ions can be simultaneously subjected to MS/MS, which significantly increases throughput compared with analyzing one ion at a time. Ion encoding of the precursor ions is accomplished by relying on the inherent mass bias associated with this ion trap when a single phase rf is applied to one pair of rods during ion accumulation. Previous work demonstrated the efficacy of a similar approach on a 3D bench top quadrupole ion trap [13]. This multiplexing advantage is particularly well suited for samples that have a transient time profile and the speed of analysis is important. Our approach allows the analysis of complex mixtures to be performed in situ while minimizing technical aspects associated with additional separation 
equipment. It is possible, however, to also use our multiplexing approach in conjunction with an up-front separation technique. Even in high-quality separations, samples may still be too complicated to obtain all of the desired information in just one run. Species that either co-elute or elute with similar time profiles may require multiple separations to acquire all of the MS/MS information. Therefore, the implementation of our multiplexed MS/MS technique should have relevance even upon further advances in miniaturized chromatography. The incorporation of a discontinuous atmospheric pressure interface (DAPI) [12] into miniaturized ion traps is an improvement in the design and capability of such instruments over previous internal electron impact (EI) ionization [1], but the DAPI source suffers from a relatively low duty cycle. The implementation of a multiplexed MS/MS method has even greater significance on systems that utilize a DAPI source as high speed MS/MS analysis can be performed. In this manuscript, we demonstrate that a miniature RIT can be successfully used to obtain tandem mass spectra for up to five compounds in only two spectra.

\section{Experimental}

\section{Instrumentation}

All experiments were carried out in a miniature rectilinear ion trap (RIT) mass spectrometer (Mini 10) constructed at Purdue University (West Lafayette, IN, USA). Operation of this device was the same as described previously [1]. Ions were generated using a homebuilt electrospray ionization (ESI) source that uses a $3 \mathrm{kV}$ potential and $\mathrm{N}_{2}$ as a nebulizing gas. Stored waveform inverse Fourier transform (SWIFT) waveforms for ion isolation and dissociation were generated by the onboard waveform generator and applied between the $\mathrm{x}$ electrodes. Ion dissociation was induced via collisions with background air.

\section{Reagents}

Serine, glutamic acid, histidine, phenylalanine, and the peptides GG, GGH, and GGGG were obtained from Sigma Aldrich Chemical Co. (St. Louis, MO, USA). Tryptophan was obtained from Fisher Scientific Co. (Fair Lawn, NJ, USA), tris(hydroxymethyl)aminomethane (tris) from EM Science (Gibbstown, NJ, USA), and the peptide GAY from American Peptide Co., Inc. (Sunnyvale, CA, USA). All compounds were dissolved in 50:50 methanol/water with $3 \%$ acetic acid to concentrations of $50 \mu \mathrm{M}$.

\section{MS/MS Conditions}

Ion isolation and dissociation were accomplished using broadband waveforms with experimentally determined frequency notches and variable peak-to-peak amplitudes. As a set of control experiments, each individual compound was subjected to MS/MS using the following conditions. Ions were first isolated with a broadband waveform for $50 \mathrm{~ms}$ and then dissociated for $20 \mathrm{~ms}$ using a dissociation voltage between 1.1 and $1.3 \mathrm{~V}$, depending on the ion of interest. In performing the multiplexed MS/MS experiments, a broadband isolation waveform incorporating frequency notches for all the ions of interest was applied for $50 \mathrm{~ms}$, and then a broadband comb dissociation waveform, incorporating the necessary frequencies, was applied for $20 \mathrm{~ms}$ at $1 \mathrm{~V}$. The existing waveform generator capabilities limited the number of multiplexed ions to five.

\section{Results and Discussion}

Three-dimensional quadrupole ion traps have an inherent mass bias that limits the $\mathrm{m} / \mathrm{z}$ range of ions that can be efficiently trapped from external ionization sources. This range can be controlled by the LMCO chosen during ion accumulation, which is set by the main rf voltage applied to the ring electrode. A similar mass bias arises on RITs when only a single phase $\mathrm{rf}$ is applied to one pair of electrodes as is the case on the miniature ion trap used in the experiments described here. Unlike RITs with rf signals applied $180^{\circ}$ out of phase on adjacent electrodes, a single-phase rf creates a strong axial rf component that ions experience upon injection into the trap. Changing the amplitude of this single-phase rf signal (or the LMCO) during accumulation of externally injected ions produces different spectra for a particular set of ions. For example, if the LMCO is increased from 30 to 40 during ion injection of a mixture of eight compounds, some ions will decrease (serine), increase (phenylalanine, tryptophan), or stay the same (GG, glutamic acid) in intensity (Figure 1). Other peptide ions (GGGG, $\mathrm{GGH}$, and GAY) start to appear in the spectrum with a LMCO of 40, whereas they are absent when the LMCO is 30. When the normalized ion intensities of these ions are plotted over a range of LMCO values during ion injection, a Gaussian-like distribution is obtained, as is illustrated for serine in Figure 2. This Gaussian-like behavior has been seen before on 3D traps [13-16], but the ion accumulation profile is slightly different. The presence of two "black holes," where ion accumulation does not occur, are observed around $\beta_{\mathrm{x}}$ values of 0.28 and $0.40 \pm 0.04$ for serine and for every ion externally ejected into the miniature RIT, and they are likely the result of ion interactions with higher order nonlinear multipole resonances. We are currently investigating these resonances as they do not correspond to typical nonlinear resonances for hexapolar or octopolar fields.

The Gaussian-like relationship between ion intensity and LMCO is reproducible for all externally injected ions, but the centers and widths of these distributions change as a function of ion $\mathrm{m} / \mathrm{z}$ ratio. If the black holes are removed, the data can be fit with a Gumbel distribution (1) that is similar in form to a Gaussian distribution but accounts for the 


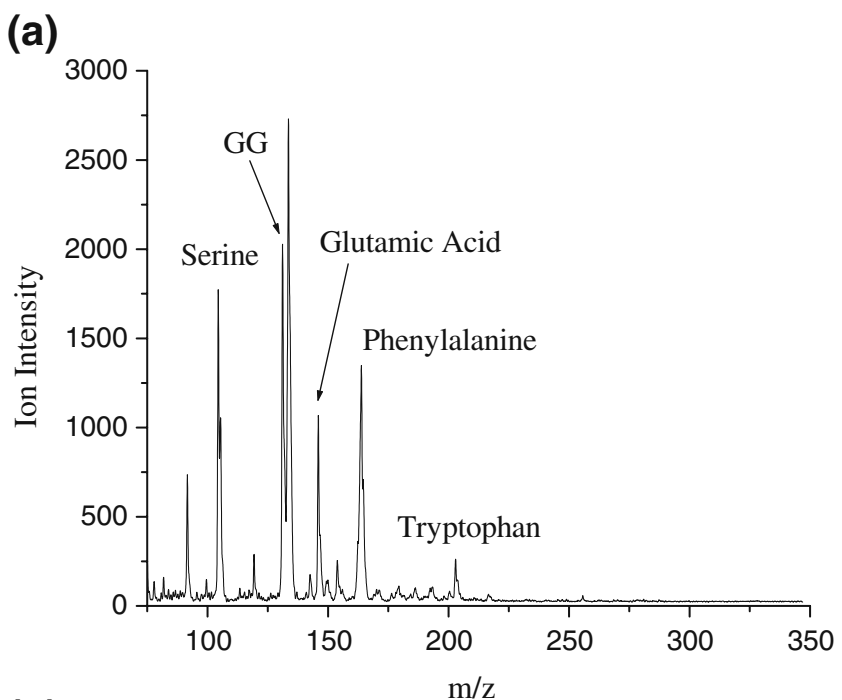

(b)

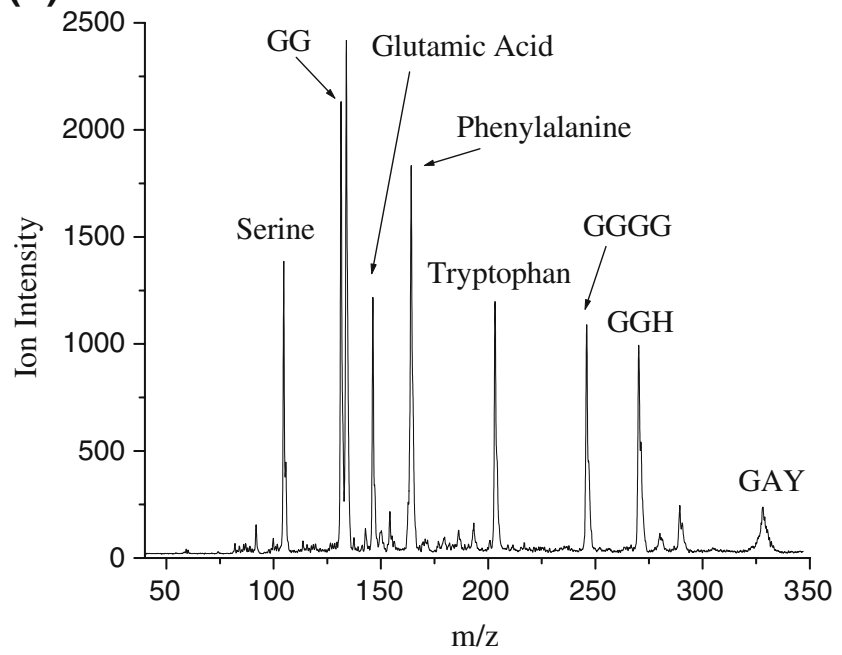

Figure 1. Mass spectra of an eight-peptide sample, showing the inherent mass bias associated with external ion accumulation on the Mini 10. Ion accumulation (a) at a LMCO of 30 and (b) at a LMCO 40

possibility of multiple Gaussians with similar, but not identical, profiles.

$$
y=A e^{-e^{\frac{-\left(x-x_{c}\right)}{w}}-\frac{x-x_{c}}{w}+1}
$$

In (1), $\mathrm{x}$ is the LMCO used for ion injection, $\mathrm{x}_{\mathrm{c}}$ is the center of the distribution (i.e., the optimum LMCO for ion accumulation), $\mathrm{w}$ is the width of the distribution, and $\mathrm{A}$ is the amplitude of the distribution, which can be normalized to 1. Upon considering the distributions for peptide ions having a range of $m / z$ ratios (Figure 3), we find the following trends. First, the distribution center (i.e., the optimum LMCO during ion accumulation) increases with increasing $\mathrm{m} / \mathrm{z}$ ratio. Second, the distribution width, as represented by one standard deviation, also increases with increasing $\mathrm{m} / \mathrm{z}$ ratio. Both the optimum LMCO as a function of $\mathrm{m} / z$ (Figure 4)

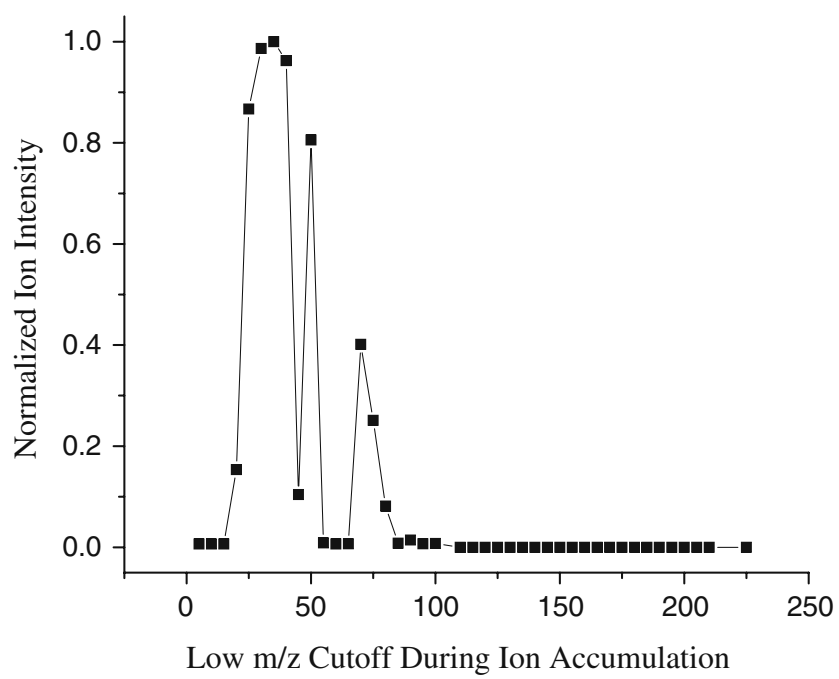

Figure 2. Normalized ion intensity of serine as a function of LMCO

and distribution width as a function of $m / z$ (Figure 5) can be plotted for a series of peptides. We hypothesize that the optimum LMCO for efficient ion trapping occurs because there is an optimum pseudopotential well depth for efficient ion trapping under a given set of ion injection conditions. To test this idea, we first rearranged the equation for pseudopotential well depth (2) in terms of $q_{x}$, producing (3).

$$
\begin{aligned}
D_{x} & =\frac{m q_{x}^{2} \Omega^{2} x_{0}^{2}}{16 e} \\
q_{x} & =\sqrt{\frac{16 e D_{x}}{m \Omega^{2} x_{0}^{2}}}
\end{aligned}
$$

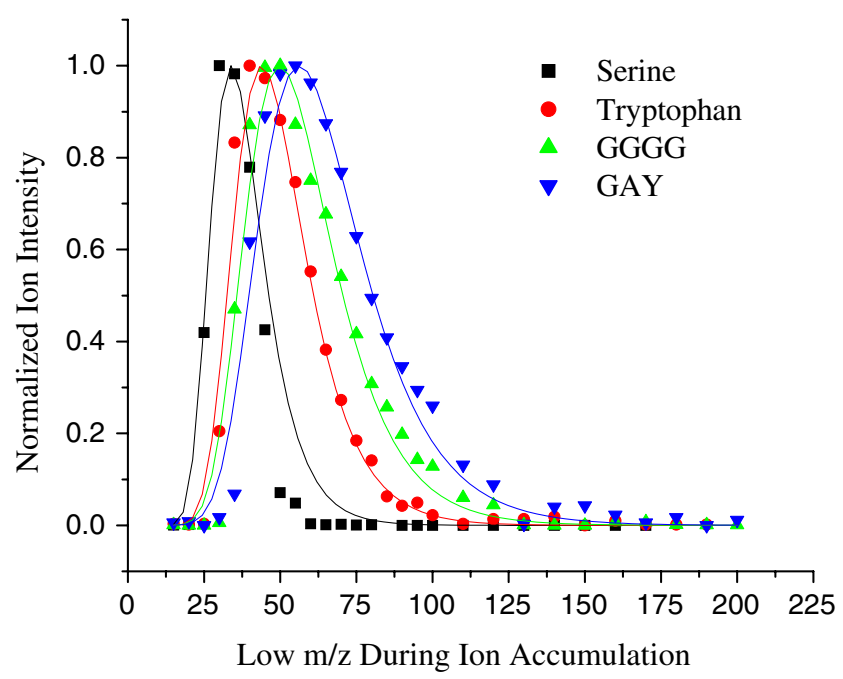

Figure 3. Normalized ion intensity of four peptide ions of increasing $\mathrm{m} / \mathrm{z}$ ratio as a function of LMCO value with "black hole" regions removed and fit with a Gumbel distribution 


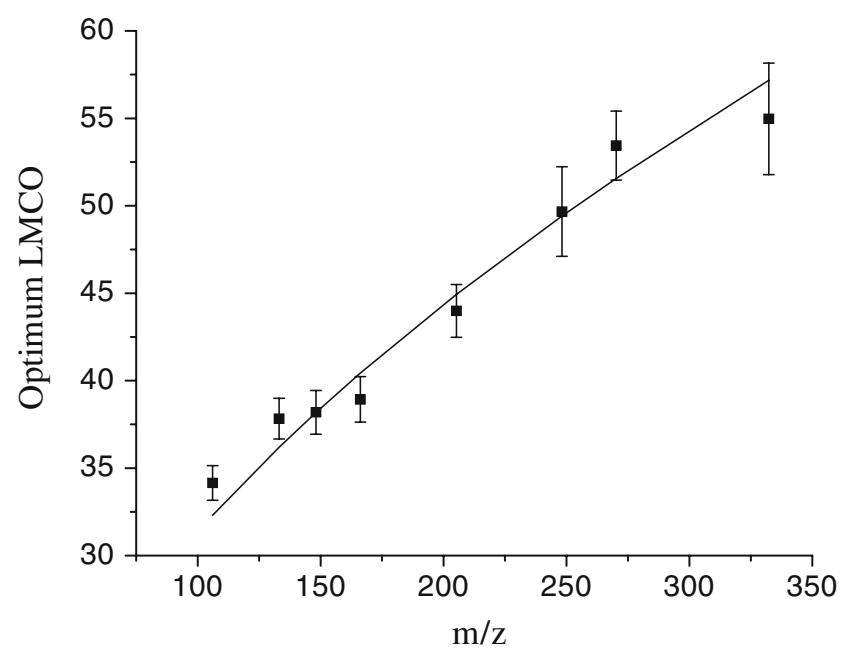

Figure 4. Optimum $L M C O$ as a function of $\mathrm{m} / \mathrm{z}$

Since $\mathrm{q}_{\mathrm{x}}$ can be written as the product of $(\mathrm{LMCO} / \mathrm{m} / \mathrm{z})$ and 0.908 , (3) can be modified in terms of LMCO (4), which now describes the relationship between ion injection LMCO and $\mathrm{m} / z$.

$$
L M C O=\frac{\sqrt{\frac{16 e D_{x}}{m \Omega^{2} x_{0}^{2}}} \times m / z}{0.908}
$$

If (4) is used to fit the experimental data, we find good agreement, suggesting that our hypothesis has some merit. The fitting parameter $\mathrm{D}_{\mathrm{x}}$ in (4) allows us to arrive at a value for the optimum pseudopotential well depth $(4.88 \mathrm{eV})$ when the equation is fit to the experimental data (Figure 4) and the standard deviation of the well depth $(0.37 \mathrm{eV}$, Figure 5).

In addition to providing some new insight into the key factor that controls efficient ion trapping of externallygenerated ions, the plots in Figures 4 and 5 allow us to develop an ion intensity encoding scheme for multiplexed MS/MS on the RIT. If a collection of ions are accumulated at one LMCO during the acquisition of a primary spectrum and then accumulated at a different LMCO during the

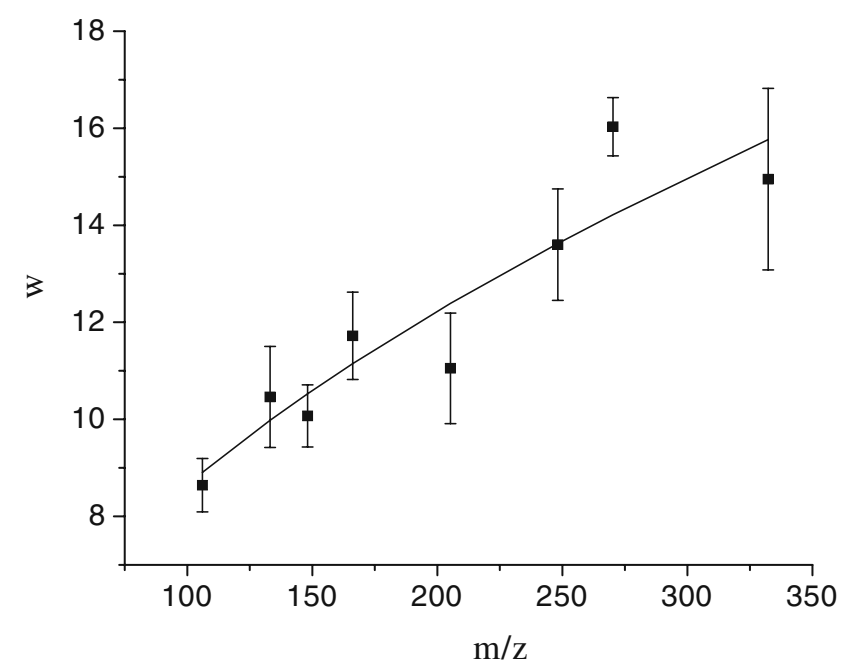

Figure 5. Ion intensity distribution widths as a function of $\mathrm{m} / \mathrm{z}$ acquisition of a secondary spectrum, the ion intensity changes can be predicted by plugging into (1) the values of $\mathrm{x}_{\mathrm{c}}$ (i.e., LMCO optimum) and w (LMCO standard deviation) obtained from (4). The ion intensity changes for each precursor ion will then apply to the ion intensity changes of all their corresponding product ions that are formed. In other words, all product ions from a given precursor ion will have a unique ion intensity ratio in going from the primary to secondary spectrum, thus permitting the product ions to be correlated back to the precursor ions from which they arose.

The application of this intensity encoding scheme is demonstrated with five ions (tris, glutamic acid, histidine, phenylalanine, and tryptophan). The procedure is as follows. First, a primary tandem mass spectrum is obtained after accumulating the precursor ions at a LMCO of 40

(a)

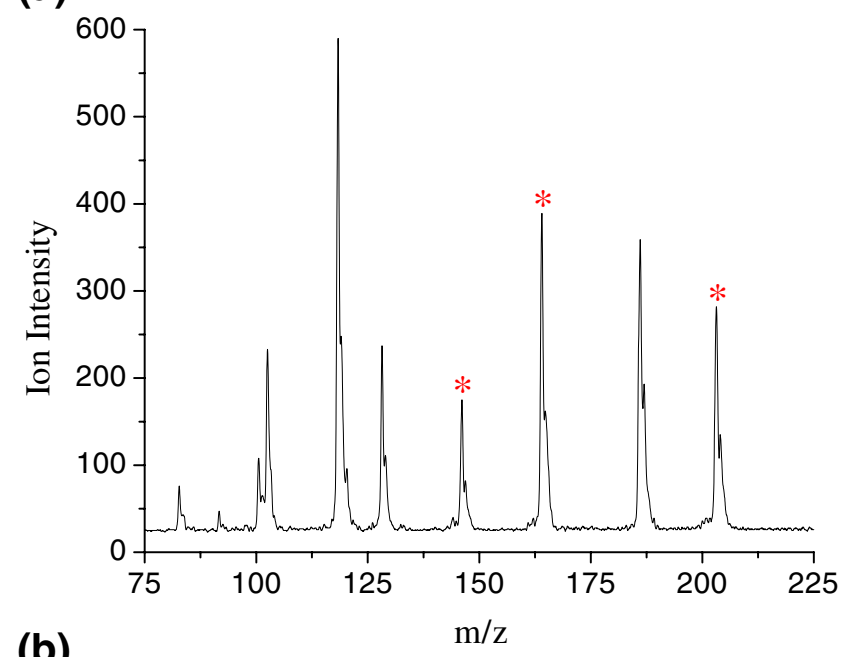

(b)

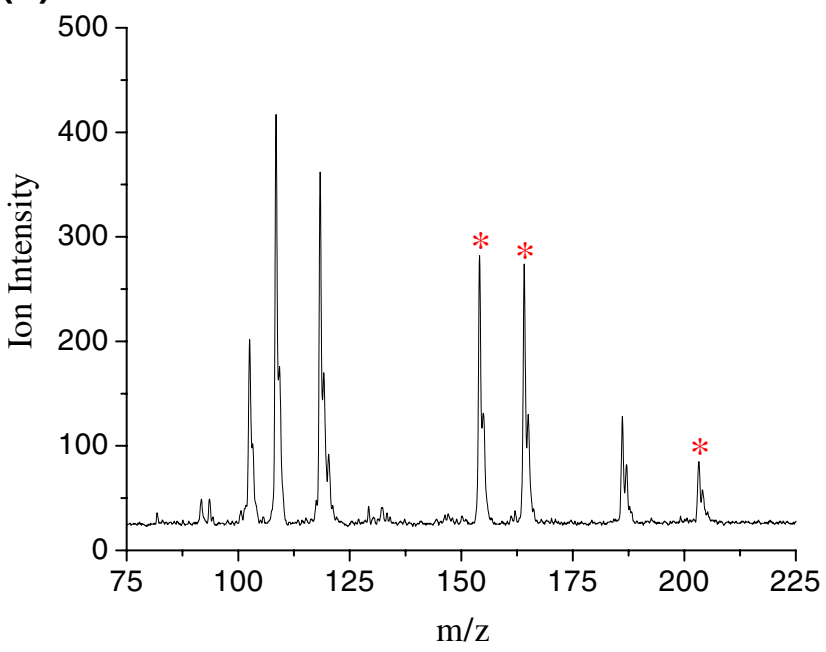

Figure 6. (a) Tandem mass spectrum of tris, glutamic acid, phenylalanine, and tryptophan accumulated at a LMCO of 30. The red asterisks indicate the presence of undissociated precursor ions. (b) Tandem mass spectrum of tris, histidine, phenylalanine, and tryptophan accumulated at a LMCO of 40. The red asterisks indicate the presence of undissociated precursor ions 


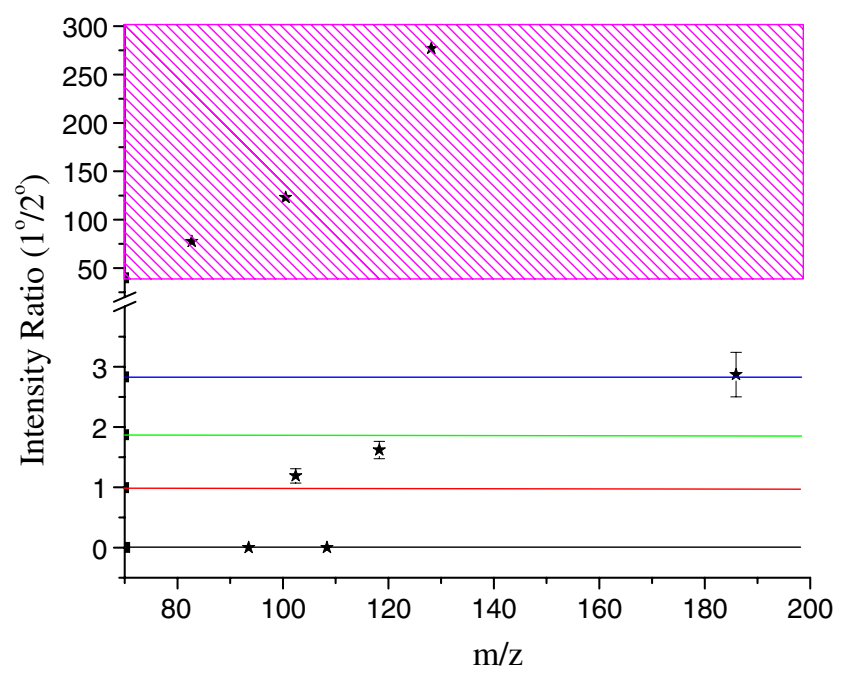

Figure 7. Product ion intensity ratios obtained by dividing the primary $\left(1^{\circ}\right)$ spectrum by the secondary $\left(2^{\circ}\right)$ spectrum. The theoretical intensity ratios are represented by a black line for histidine, a red line for tris, a green line for phenylalanine, a blue line for tryptophan, and the magenta section for glutamic acid. Observed fragment ions in the multiplexed MS/MS experiments are shown as black stars with their corresponding error bars

(Figure 6a). Acquisition of this primary spectrum involves simultaneously isolating the precursor ions and then simultaneously dissociating the precursor ions using broadband waveforms. Then, a secondary tandem mass spectrum is obtained after accumulating the precursor ions at a LMCO of 30 (Figure 6b). Again, all the precursor ions are simultaneously isolated and then simultaneously dissociated. Finally, the ion intensity ratios for each product ion are determined by dividing the intensities from the primary spectrum $\left(1^{\circ}\right)$ by the intensities from the secondary spectrum $\left(2^{\circ}\right)$. The product ion intensity ratios can be plotted (Figure 7), which helps correlate each product ion with its corresponding precursor ion. To find the correct precursor ion/product ion correlations, the observed ion intensity ratios (Figure 7) can be compared with the predicted precursor ion intensity ratios that are calculated using (1) and (4). The theoretical ion ratios for the precursor ions are: tris (1.0), glutamic acid (>100), histidine (0), phenylalanine (1.8), and tryptophan (2.8). The very large value for glutamic acid ( $>100 ; 4000$ maximum intensity/40 average noise intensity) is due to the fact that this ion was only accumulated and dissociated in the primary spectrum. A value of zero for histidine is due to the fact that this ion was only accumulated and dissociated in the secondary spectrum. The plot in
Figure 7 shows the theoretical ion ratios of each precursor ion (colored lines/areas) in conjunction with observed ion ratios of the measured product ions (stars). A more detailed listing of the information is also included in Table 1. From this data, the observed product ions can all be correctly assigned. Control experiments in which tandem mass spectra for each precursor ion is individually obtained (data not shown) confirm the product ion assignments in Figure 7 and Table 1.

There are, however, three product ions observed in the individual MS/MS experiments that are not observed in the multiplexed MS/MS experiments. Two product ions from phenylalanine $(\mathrm{m} / z 105$ and 149) are likely not observed in the multiplexed experiment because they have low abundance in the individual tandem mass spectrum. Due to incomplete precursor ion dissociation (in both the primary and secondary spectra), it is not surprising that these low intensity products are not seen. During the multiplexed MS/ MS experiments, the dissociation amplitude chosen is a compromise between the optimum values for all of the ions being dissociated. The waveform generator associated with the Mini 10 does not allow the user to create waveforms with different amplitudes over different frequency ranges. Therefore, incomplete dissociation for many of the larger precursor ions is expected as they generally require higher voltages to achieve more complete dissociation. The phenylalanine product ion at $m / z 149$ might also be absent because it has a $\mathrm{m} / \mathrm{z}$ ratio close to the $\mathrm{m} / \mathrm{z}$ ratio of another precursor ion (glutamic acid) and so, as it is formed, it might be further dissociated by the waveform used to dissociate glutamic acid. A third product ion that is not observed is $m / z 130$ from tryptophan. The reason for this is that glutamic acid also gives rise to a product ion at this $\mathrm{m} / \mathrm{z}$ ratio. Because glutamic acid has an intensity ratio $>100, \mathrm{~m} / z 130$ is encoded with its intensity ratio. Coincident ions such as these (product ions that have the same $m / z$ as a product ion from a different precursor) could be deconvoluted by acquiring a third or tertiary spectrum that would result in precursor ion intensities being changed by a different ratio.

It should be noted that the relatively few product ions that are observed in these tandem mass spectra are due to the small size of the precursor ions that were chosen. Most of the ions have very few dissociation channels. Compound choice in these experiments was dictated by the limited $\mathrm{m} / \mathrm{z}$ range $(\sim 350 \mathrm{Da})$ of the miniature ion trap used in these experiments. This limited mass range can be circumvented by improving the miniature ion trap design to minimize $\mathrm{rf}$ discharge at higher $\mathrm{V}$ or by lowering the ejection $\mathrm{q}_{\mathrm{x}}$ to extend the working $\mathrm{m} / \mathrm{z}$ range.

Table 1. Product/precursor ion identification using the multiplexed MS/MS approach

\begin{tabular}{|c|c|c|c|c|c|c|c|c|}
\hline Observed product ion $\mathrm{m} / \mathrm{z}$ & 84 & 95 & 102 & 104 & 110 & 120 & 130 & 188 \\
\hline Observed ratio & $>100$ & 0 & $>100$ & $1.2 \pm 0.1$ & 0 & $1.6 \pm 0.1$ & $>100$ & $2.9 \pm 0.4$ \\
\hline Theoretical ratio & $>100$ & 0 & $>100$ & 1.01 & 0 & 1.85 & $>100$ & 2.83 \\
\hline Correlated precursor ion & Glutamic acid & Histidine & Glutamic acid & Tris & Histidine & Phenylalanine & Glutamic acid & Tryptophan \\
\hline
\end{tabular}




\section{Conclusions}

We have demonstrated that a miniature RIT mass spectrometer is capable of performing multiplexed MS/MS. A multiplexed $\mathrm{MS} / \mathrm{MS}$ approach is advantageous for the analysis of complex mixtures in the field as separation techniques are not as readily coupled to portable MS instrumentation. To accomplish multiplexed MS/MS on the miniature ion trap, we implement an ion intensity encoding scheme in which the changes in precursor ion intensities between a primary spectrum and secondary spectrum are transferred to their corresponding product ions. This method is straightforward to implement as the ion intensity encoding scheme simply requires ion accumulation to be carried out at two different LMCO values. Such a simple encoding scheme is possible because there exists a reproducible and predictable relationship between ion intensity and the LMCO used during external ion accumulation. In this work, we demonstrated that multiplexed MS/MS could be performed on five ions by acquiring only two product ion spectra, and all the resulting product ions could be correctly correlated with their associated precursor ions. In theory, this multiplexed approach could be applied to a greater number of precursor ions, and future work will extend this approach to more complicated samples and ions with higher $\mathrm{m} / \mathrm{z}$ ratios.

\section{Acknowledgments}

The authors thank the Office of Naval Research through the DURIP program (grant N000-14-07-1-0980) for support of this work. They also thank Professor Graham Cooks, Dr. Robert Noll, Dr. Ewa Sokol, Dr. Nathan Sanders, and Dr. Guangming Huang from Purdue University for technical assistance with the Mini 10.

\section{References}

1. Gao, L., Song, Q., Patterson, G.E., Cooks, R.G., Ouyang, Z.: Handheld rectilinear ion trap mass spectrometer. Anal. Chem. 78(17), 5994-6002 (2006)

2. Smith, P.A., LePage, C.J., Lukacs, M., Martin, N., Shufutinsky, A., Savage, P.B.: Field-portable gas chromatography with transmission quadrupole and cylindrical ion trap mass spectrometric detection: chromatographic retention index data and ion/molecule interactions for chemical warfare agent identification. Int. J. Mass Spectrom. 295(3), 113-118 (2010)

3. Shortt, B.J., Darrach, M.R., Holland, P.M., Chutjian, A.: Miniaturized system of a gas chromatograph coupled with a Paul ion trap mass spectrometer. J. Mass Spectrom. 40(1), 36-42 (2005)

4. Diaz, J.A., Pieri, D., Arkin, C.R., Gore, E., Griffin, T.P., Fladeland, M., Bland, G., Soto, C., Madrigal, Y., Castillo, D., Rojas, E., Achí, S.: Utilization of in situ airborne MS-based instrumentation for the study of gaseous emissions at active volcanoes. Int. J. Mass. Spectrom. 295(3), 105-112 (2010)

5. Getty, S.A., ten Kate, I.L., Feng, S.H., Brinckerhoff, W.B., Cardiff, E. H., Holmes, V.E., King, T.T., Li, M.J., Mumm, E., Mahaffy, P.R., Glavin, D.P.: Development of an evolved gas-time-of-flight mass spectrometer for the Volatile Analysis by Pyrolysis of Regolith (VAPoR) instrument. Int. J. Mass Spectrom. 295(3), 124-132 (2010)

6. Smith, J.N., Keil, A., Likens, J., Noll, R.J., Cooks, R.G.: Facility monitoring of toxic industrial compounds in air using an automated, fieldable, miniature mass spectrometer. Analyst 135(5), 994-1003 (2010)

7. Lauritsen, F.R., Nielsen, K.: Fast and direct recognition of the active ingredients in tablets using hot cell membrane inlet mass spectrometry. Int. J. Mass Spectrom. 295(3), 119-123 (2010)

8. Ouyang, Z., Cooks, R.G.: Miniature mass spectrometers. Annu. Rev. Anal. Chem. 2, 187-214 (2009)

9. Meuzelaar, H.L.C., Dworzanski, J.P., Arnold, N.S., McClennen, W.H., Wager, D.J.: Advances in field-portable mobile GC/MS instrumentation. Field Anal. Chem. Tech. 4(1), 3-13 (2000)

10. Contreras, J.A., Murray, J.A., Tolley, S.E., Oliphant, J.L., Tolley, H.D., Lammert, S.A., Lee, E.D., Later, D.W., Lee, M.L.: Hand-portable Gas Chromatograph-Toroidal Ion Trap Mass Spectrometer (GC-TMS) for detection of hazardous compounds. J. Am. Soc. Mass Spectrom. 19(10), 1425-1434 (2008)

11. Ouyang, Z., Wu, G., Song, Y., Li, H., Plass, W.R., Cooks, R.G.: Rectlinear ion trap: concepts, calculations, and analytical performance of a new mass analyzer. Anal. Chem. 76(16), 4595-4605 (2008)

12. Gao, L., Cooks, R.G., Ouyang, Z.: Breaking the pumping speed barrier in mass spectrometry: discontinuous atmospheric interface. Anal. Chem. 80(11), 4026-4032 (2008)

13. Wilson, J., Vachet, R.W.: Multiplexed MS/MS in a quadrupole ion trap mass spectrometer. Anal. Chem. 76(24), 7346-7353 (2004)

14. McLuckey, S.A., Glish, G.L., Asano, K.G.: Coupling of an atmospheric-sampling ion-source with an ion-trap mass-spectrometer. Anal. Chim. Acta 225(1), 25-35 (1989)

15. Louris, J.N., Amy, J.W., Ridley, T.Y., Cooks, R.G.: Injection of ions into a quadrupole ion trap. Int. J. Mass Spectrom. Ion Processes 88(2/ 3), 97-111 (1989)

16. Kofel, P.: In: March, R.E., Todd, J.F.J. (eds.) Practical Aspects of Ion Trap Mass Spectrometry, vol. II, pp. 51-87. CRC Press, New York (1995) 